\title{
Introduction to the Constellations
}

\section{EXHIBIT: $\quad$ Galileo's World}

GALLERY: Controversy over the Comets; Music of the Spheres

The Sky at Night; Space Science after Galileo

OBJECT: $\quad$ Urania's Mirror (London 1825), a boxed set of 32 cards; with

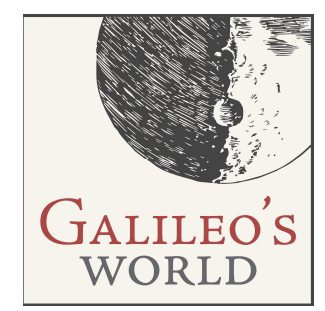
Jehoshaphat Aspin, A Familiar Treatise on Astronomy

(London 1825), $2 \mathrm{~d}$ ed., or any celestial atlas.

Choose one of the constellation cards from Urania's Mirror and hold it up to the light.

Compare the star patterns (where light comes through the holes) with the constellation figures (drawn on the front side of the cards).

\section{Q. What is the difference between star patterns and constellation figures?}

A. The star patterns are the configuration of the stars in the sky; the constellation figures are the imaginary pictures that help us identify the stars.

\section{Q. Do the star-patterns of constellations resemble the figures they are named for?}

A. No. The stars are named and grouped into recognizable clusters or constellation figures as an aid to memory. Constellation patterns are arbitrarily imposed upon the stars in honor of particular characters or stories, not because the pattern resembles the character in form.

Example: Only the most imaginative can see a bear in the area of the Big Dipper. There are many star-patterns that look like dippers, triangles, or squares in the sky. There are very few that actually resemble bears or people. Yet constellations were named to honor particular figures in ancient stories, not because the star pattern actually looked like the figure being honored. It took imagination to invent them a long time ago, and it takes imagination to see them now. The "handles" of the dippers represent the "tails" of the bears on ancient star maps--even though modern bears don't have long tails! The Big Bear was regarded as a bear before Homer, and an ancient Greek story explains why the Big and Little Bears have such long tails.

\section{Q. How many constellations are there?}

A. As many as you can imagine! 88 constellations are officially recognized today, and many of these are of ancient origin. Others, especially in the southern hemisphere, date from more recent times. But every culture tells stories about the sky, and passes its skylore down from generation to generation with many constellation figures. You can do this, too.

\section{Q. What is the difference between a constellation and an asterism?}

A. An asterism is a star-pattern that is not a constellation. Asterisms may be contained within a single constellation or consist of stars belonging to different constellations. The Big and Little Dippers are asterisms within the constellations of Ursus Major and Ursus 
Minor. The Summer Triangle and Winter Hexagon are asterisms that include stars from several constellations.

\section{Activity}

1. Choose one of the constellation cards from Urania's Mirror and obtain a printed copy on cardstock.

2. Compare the style and depiction of the constellation figure on your cardstock with illustrations of the same constellation from other star atlases.

What similarities and differences do you see?

Which are your favorite depictions of this constellation?

3. With crayons or permanent markers, color your constellation.

4. Place your colored card on top of a blank sheet of cardstock, and then place both sheets on a hard surface to use as a cutting board. With a sharp instrument, poke holes through the bright stars of both the colored constellation card and the blank sheet of card stock.

5. Hold up the colored constellation card to the light. Compare the star pattern visible through the punched holes with the constellation figure.

6. Think of one of your favorite stories. It can be a story that really happened, in history or with you or your family, or it can be an imaginative story from a book or a movie or one that you make up yourself. Choose a figure from that story and draw it around the holes that were punched in the blank sheet of card stock. Be creative. Name some of the stars as you wish ("armpit of the mighty one," etc.). Color your new constellation.

7. Share your new constellation and star names with others. Look for it in the actual sky next time you are outdoors under the starry night!

Provided by Lynx Open Ed Collaborating in exhibit-based learning, lynx-open-ed.org

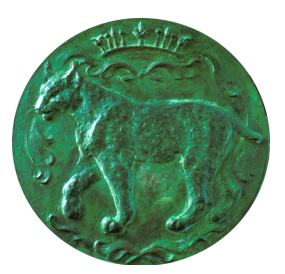

